\title{
Design and Experimental Validation of Radio Access Network Controller Prototype for Multi-RAT Technologies with Scheduler Strategies
}

This paper was downloaded from TechRxiv (https://www.techrxiv.org).

\section{LICENSE}

CC BY-NC-SA 4.0

SUBMISSION DATE / POSTED DATE

08-10-2021 / 13-10-2021

\section{CITATION}

Rahman, Md Arifur; Flizikowski, Adam; Pietrzyk, Slawomir; Mowla, Md Munjure (2021): Design and Experimental Validation of Radio Access Network Controller Prototype for Multi-RAT Technologies with Scheduler Strategies. TechRxiv. Preprint. https://doi.org/10.36227/techrxiv.16769422

$\mathrm{DOI}$ 


\title{
Design and Experimental Validation of Radio Access Network Controller Prototype for Multi-RAT Technologies with Scheduler Strategies
}

\author{
Md Arifur Rahman, Adam Flizikowski, Slawomir Pietrzyk, Md Munjure Mowla \\ IS-Wireless, Pulawska Plaza, ul. Pulawska 45b, 05-500 Piaseczno, Poland \\ a.rahman@is-wireless.com; a.flizikowski@is-wireless.com; s.pietrzyk@is-wireless.com; m.mowla@is-wireless.com
}

\begin{abstract}
Future wireless networks will be more heterogeneous due to different radio access technologies (RATs), types of interconnecting links between the users, and diverse requirements of the connected users. The multiple RAT (multi-RAT) network composed of cellular and Wi-Fi access points is considered as a promising solution to meet indoor traffic needs during pandemic situations. Therefore, in this paper we design and provide an experimental validation of a radio access network controller (RANC) prototype solution and investigate different long term evolution medium access control (LTE MAC) scheduling strategies to show the quality-of-service $(\mathrm{QoS})$ and quality-ofexperience $(\mathrm{QoE})$ performance of multi-RAT. The main aspects of this paper are to show the involvement towards coordination strategies amongst multiple RATs and integration of parametric control of higher MAC and upper layer network protocols. Moreover, we also evaluate and examine the possibility to take advantage of the fact of using interworking concepts such as lightweight Internet protocol (LWIP) and LTE-WLAN aggregation (LWA) while making the scheduling decisions. Such a solution would contribute to the software-defined networking (SDN) approach, where multi-RAT aware scheduler adapts to dynamic channel conditions to provide robustness against severe real-time channel conditions. Finally, we provide comparative analysis of multiRAT scenarios and evaluate the QoE performance of different scheduling algorithms with SINR based information centric LWA switching and QoE-aware LWA switching by using RANC.

Index Terms-Radio access network controller, softwaredefined networking, lightweight internet protocol (LWIP), LTEWLAN aggregation, and quality-of-experience (QoE).
\end{abstract}

\section{INTRODUCTION}

$\mathbf{T}$ HE COVID-19 pandemic outbreak was an extreme exercise for all mankind but in particular it put large stress on the professional life i.e., employers, employees and especially various kinds of network providers and Internet service providers (ISPs) due to "home office". It has provided a global field trial and a stress test for the whole network ecosystem. The main professional activity of most employees around the world has shifted to the "home office" with tele-work. In parallel the younger family members have also been shifted to distance learning mode for participating virtual classes. As a result (a) cable-based access networks (e.g. CATV) have received a large share of load during working hours as well as (b) mobile network operators (MNO) who also assured their infrastructure for remote access to content and connectivity. The number of teleconferences run through multiple platforms skyrocketed as it became the replacement for face-to-face communications. This way houses became real network test playground, where intensively some members engaged in teleconferences (parents for works, kids for classes), others played online games or watched videos in parallel.

This paper is mainly zoomed on the traffic profiling during COVID-19 pandemic outbreaks and propose a robust and novel solution based on SDN-driven switching of downlink traffic between two different radio access technologies (RAT) - so called multi-RAT. However, to schedule the resources in multi-RAT technologies a number of research works have been investigated in recent years [1-6]. In [1], the authors propose a multi-path based adaptive concurrent transfer scheme that allows application layer traffic transmission via user datagram protocol (UDP). The proposed method can improve the throughput of the system while satisfying the constraints of quality-of-experience $(\mathrm{QoE})$, the video encoding rate, and the priority of the frame, respectively. Similarly, in a multiRAT environment the coordination among different RATs, the coordinated architecture of the RAT, and resource management policies along with a packet-level RAT selection and control algorithm is proposed by Jihan et al. [2] to evaluate throughput and energy consumption performance of the underlying RAT. Multi-RAT connectivity in heterogeneous networks and a riskaverse traffic allocation scheme is proposed in [3] that provides a trade-off model of expected throughput to measure the risk of semivariance of the throughput. Fast-RAT scheduling solution in a $5 \mathrm{G}$ multi-RAT scenario is studied by Victor $e t$ al. [4] where reference signals received power (RSRP), received signal strength indication (RSSI), and reference Signal Received Quality (RSRQ) based criteria are used to select the suitable RAT. It has been also validated in the paper that the RSRP-based RAT selection provides better user equipment (UE) throughput performance than other methods used for simulation comparison.

As is known, the LTE-WLAN aggregation (LWA) combines the resources of both RAT's and provide us the opportunity of sharing both LTE and Wi-Fi capacities. In this mechanism, it is very important that the network should intelligently steer the traffic between two different RATs to satisfy the QoE of the users. Similarly, the LTE-WLAN integration (LWIP) with IPSec tunnel is a third-generation partnership project (3GPP) feature that can enable optimal integration of Wi-Fi with LTE access network. In a series of works [7-9], Afaqui 
et al. showed the design mechanisms and implementation of these (LWA and LWIP) mechanisms through National Instrument software-defined radio (SDR) setup at the Online Wireless Laboratory of Technical University Dresden (TUD). In the paper, we integrate the experimental setup inside the TUD setup that have been defined by orchestration and reconfiguration control architecture (ORCA) consortium [10] to access the platform. For this research, we use the TUD platform within the scope of ORCA project that allow us to implement and test a prototype of radio access network controller (RANC) for a multi-RAT environment which can enable the LWA and LWIP concepts to steer the traffic by maximizing the utility of multimedia end-user while aggregating and dynamically switching traffic between Wi-Fi and LTE. In multi-RAT environments, cell association, radio resource scheduling mechanism, mode selection, and multi-connectivity scheduling solutions have already been investigated to evaluate the throughput performance of the networks [3], [5], and [6]. However, to the best of author knowledge, this paper firstly explores a prototype of a RANC for a multi-RAT environment and evaluates the performance of predicted QoE. The key contributions of the paper are summarized as follows:

- Firstly, we implement and test a prototype of RANC in a multi-RAT environment within the scope of TUD testbed. The prototype of RANC can take over the decision by enabling LWA and LWIP in a multi-RAT environment that can be further communicated with the LTE MAC scheduler to enhance the efficiency of scheduling decisions. We also evaluate the possibilities of adopting a RANC from a LTE scheduler perspective to support single (LTE) or multiple (LTE and Wi-Fi) technologies.

- Secondly, we verify and analyze the potential migration of the MAC scheduler from single RAT to multi-RAT technologies and analyze the RANC requirements to understand the LWA and LWIP mechanism while identifying the potential issues for multi-RAT deployment for future wireless networks.

- Finally, we provide comparative analysis of multi-RAT scenarios based on LWA and LWIP concepts that will evaluate the performance of Round Robin scheduler in only LTE access network without RAN Controller (denoted as RR-LTE), the LTE access network only with Proportional Fair scheduler without RAN controller (denoted as PF-LTE), the SINR based information centric LWA switching from LTE to Wi-Fi using a RANC, and the QoE-aware LWA switching from LTE to Wi-Fi via RAN controller, respectively.

The rest of the paper is organized as follows. A System model for experimental validation is addressed in Section II. Section III discusses the multi-RAT activation mechanism and functional architecture of multi-RAT activation through RANC. Section IV illustrates the experimental framework and experimental execution process. Section V provides performance analysis of the conducted experiment. Finally, conclusion and future research direction are discussed in Section VI.

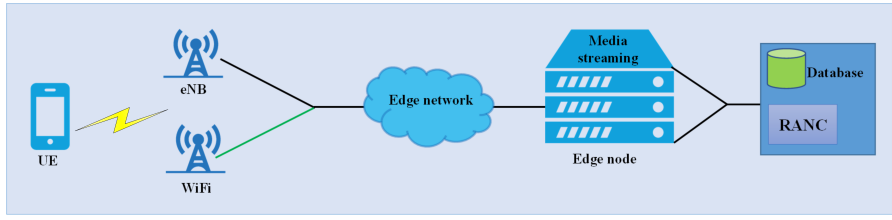

Fig. 1. System Model.

\section{SySTEM MODEL}

We consider a multi-RAT activation framework as a system model where a user coexist with two different RATs. As shown in Fig. 1, the UE will collect the Quality of Service/Quality of Experience (QoS/QoE) Key Performance Indicators (KPIs). During experiments, signal to interference plus noise ratio (SINR) is considered as a QoS-like KPI and the Internet Protocol Television (IPTV) streaming quality parameters (video resolution, playback bit rate, frame rate, the packet loss frequency, and frame loss frequency) as a QoEbased KPI. The aforementioned information is collected by the UE and sent to the so-called specific monitoring agent called "testman server" (a dedicated instrumentation solution) through the evolved Node B (eNB) and it is stored in the database of the RANC entity.

The RANC retrieves this information from the database and evaluates according to the predicted objective function (predicted QoE). If the predicted QoE criteria is not satisfied, the RANC performs a LWA and LWIP mechanism between LTE and Wi-Fi to meet the predicted QoE. In the experiments, the criteria of activating LWA and LWIP mechanism is based on the SINR or the QoE measurements depending on the scenarios. The RANC for multi-RAT activation is implemented in Python, which performs the LWA and LWIP mechanisms between the LTE and Wi-Fi links. The RANC is composed of multiple applications for the network monitoring and management. The implemented RANC module is composed of several different RAN controller applications which will perform the functionalities of monitoring of SINR via testman client, CSV $\log$ of the monitored SINR for further data analysis, and network management. In the proposed experimental validation, the LWA and LWIP mechanisms are executed based on the monitored SINR, monitoring of QoE influence factors of the IPTV transmission via MongoDB, QoE measurements of the IPTV transmission using QoE model presented in [11]. The traffic is steered between the LTE and Wi-Fi based on the monitored QoE KPIs of TUD experimental platform.

\section{Multi-RAT Activation Through RANC}

In the paper, we assume that a single slice is already given but its corresponding RAT is switched based on the collected QoS/QoE measurements information from the user and the traffic. The multi-RAT activation through RANC will allow the user to receive services from different RATs based on the reported QoS/QoE measurement information to the RANC. At the beginning of the multi-RAT activation procedure, the 


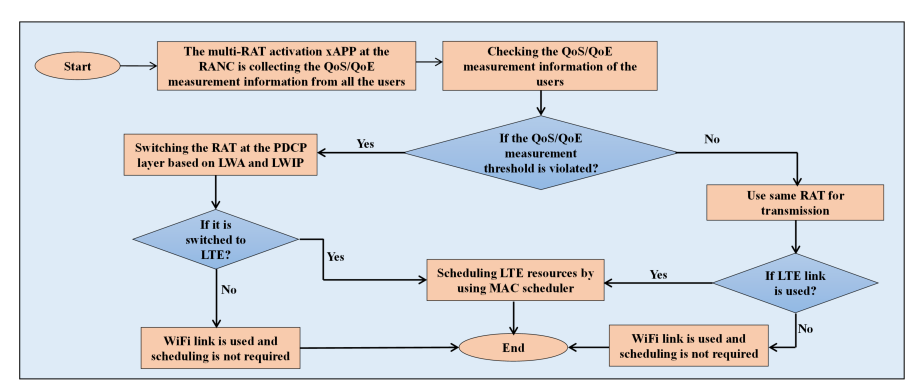

Fig. 2. The multi-RAT activation procedure through RANC.

application at the RANC will be collecting the QoS/QoE measurements information from the user. The RANC application will perform the SINR monitoring in real-time for the LTE link and it will also create a CSV $\log$ file of the measured SINR. Once the SINR information is available at the RANC it will perform LWA and LWIP operation-based switching/spliting based on the real-time value of the SINR, e.g., if the SINR value is less than $10 \mathrm{~dB}$, the RANC will perform an action to steer traffic to Wi-Fi link. On the other hand, the video streaming client at the UE is also monitoring the QoE KPI and sending the information to the MongoDB database and it is assumed that the database is available at RANC for collecting the measurement information. The RANC application of multiRAT activation can perform the QoE monitoring in real-time by collecting updated QoE KPI's from the MongoDB database. Similarly, the application can also generate local CSV log files of the measured QoE.

As shown in Fig. 2, the switching of the RAT will only happen, if the measured QoS/QoE is violated the given threshold. If the threshold of QoS, i.e., the measured SINR of the LTE link is violating a given threshold, it will switch to Wi-Fi link. However, if it is not violating the threshold it will use the LTE link, and the MAC scheduler at eNB will schedule resources to the user. Similarly, in the case of QoE KPI of IPTV video streaming, if the actual QoE measurement is also violating a given threshold, it will switch from one RAT to another. In the traffic switching stage, if the traffic is steered to LTE link then, the eNB MAC scheduler will schedule resources to the user, otherwise Wi-Fi link will be used and the video streaming will be delivered to the user. The detailed implementation of the RANC module is composed of several applications and execute following functionalities, e.g., monitoring of SINR via testman client CSV $\log$ of the monitored SINR for further data analysis, network management by enabling LWA and LWIP and switching based on the monitored SINR, monitoring of QoE influence factors of the IPTV transmission via MongoDB, QoE measurements of the IPTV transmission using QoE model presented in [11], and the QoE-aware network management using LWA and LWIP enabling and steer traffic based on monitored QoE KPIs, respectively. The experimental validation of multi-RAT activation through RANC will be discussed in detail in Section IV.

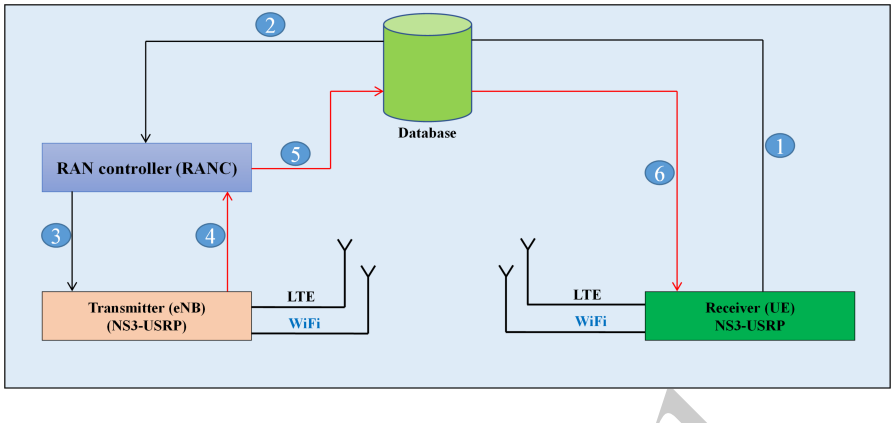

Fig. 3. Functional Architecture of Multi-RAT Activation Through RANC.

\section{A. Functional Architecture of Multi-RAT Activation}

The proposed architecture follows the information-centric functional architecture for the network monitoring and management in the multi-RAT environment. The proposed architecture is inspired by the work proposed in [12] where the probe installed at the UE terminal provides QoS/QoE KPIs to the controller to perform network management operations. The proposed architecture as shown in Fig. 4, the user-end probe will collect the QoS/QoE KPIs. In the paper, we consider SINR as QoS and IPTV streaming as a QoE KPI-ss that will include video resolution, playback bitrate, frame rate and packet loss frequency/frame loss frequency. The aforementioned information is collected by the UE probe and stored in the database that is accessible by the RANC on regular time intervals e.g., the UE sends the collected information after every 1 second to the database. The RANC accesses this information from the database and evaluates according to the objective function. If the objective function criteria is not satisfied, the controller performs LWA and LWIP procedures between the LTE and Wi-Fi to meet the objective criteria. Moreover, the customized LTE MAC scheduler is a part of the experiments which run in all the scenarios. The following information is being exchanged among the modules which is shown in Fig. 3 and the functionalities of each step is described as follows:

- Feedback from the UE: The UE probe sends the QoE related KPIs to the database.

- Retrieval of KPIs: The RAN controller retrieves the QoE related KPIs from the database.

- Control Operation: The RAN controller performs the control action based on the measurement criteria

- Acknowledgement from the base station after performing control action

- Acknowledgment from controller to the database that control action has been performed

- Acknowledgment from the database to the UE that control action has been performed

We consider the IPTV video transmission as a use case and we also validate these in the later section of the paper. The IPTV media streaming is implemented via VLC in Python Language. In the current implementation, the media client 
receives the stream from the media server. The user-end probe implemented at the client side collects the QoE KPIs. The IPTV media streaming module is composed of Media Server and Client. In the Media Server the Media files are placed in the media server which will broadcast the IPTV transmission using UDP legacy protocol at the network transport layer. Moreover, the Media Client will perform the IPTV media streaming from the media streaming server over UDP protocol. It will also collect the media streaming session information (QoE KPI) via passive user-end probe for QoE monitoring. Additionally, it will also store the QoE KPI in the database on the regular intervals based on the monitoring frequency. The Media Client generated CSV logs of the monitored QoE KPI's for further analysis.

\section{EXPERIMENTAL VALIDATIONS}

In this section, we provide the details of the validation of multi-RAT activation through RANC. The experiments are performed 5 times i.e., number of runs are 5 per scenario. In the experiments, we use a two minutes video sequence of Big Buck Bunny which is encoded in H.264 having HD 1080p resolution and 30 frames per second frame rate. The IPTV streaming based on UDP protocol is started from the media server to the media client over the air interface. For all the experiments, we have utilized Additive White Gaussian Noise (AWGN) at frequency $2 \mathrm{GHz}$ with the $2 \mathrm{dBm}$ output power and $10 \mathrm{M}$ samples/s is generated by the interferer setup. The generated noise by the interferer is coupled with the LTE link in the testbed setup by combiner in the downlink direction. The generated noise by the interferer varies the SINR in the range $12-14 \mathrm{dBs}$ at the LTE link. For the RAN controllerbased scenarios to monitor the QoE and SINR, the monitoring frequency of the probe is kept to $1 \mathrm{~Hz}$ (1 second sampling interval) in all the runs. For the LTE link, we consider Round Robin and Proportional Fair schedulers at the MAC layer. To compute the QoE of IPTV streaming, we utilized the QoE Model proposed in [11] and it is defined as follows:

$$
Q o E=1+\left(v_{1}-\frac{v_{1}}{1+\left(\frac{B R}{v_{2}}\right)^{v_{3}}}\right) \exp \left(-\frac{P L F}{v_{4}}\right)
$$

where $v_{1}=3.8, v_{2}=4.9, v_{3}=3.6$, and $v_{4}=3.5$ are the coefficient model while $B R$ and $P L F$ are the source coding rate of the video and the packet loss rate of the network, respectively. To validate the multi-RAT activation through RANC, we have carried out an experiment by taking an advantage of link diversity (LTE or Wi-Fi) in the system. The system initiates the transmission using LTE under the continuous control from RAN. The RANC takes the responsibility of switching traffic between the links based on the SINR. In addition, one of the customized scheduling algorithms is configured in LTE in order to exploit fading conditions. The SINR is made available at the RANC through a testmen server that enables sending the SINR value to testment client, as shown in Fig. 4. In order to leverage the multi-RAT testbed, intelligent RAN switching can be a viable design for testbed

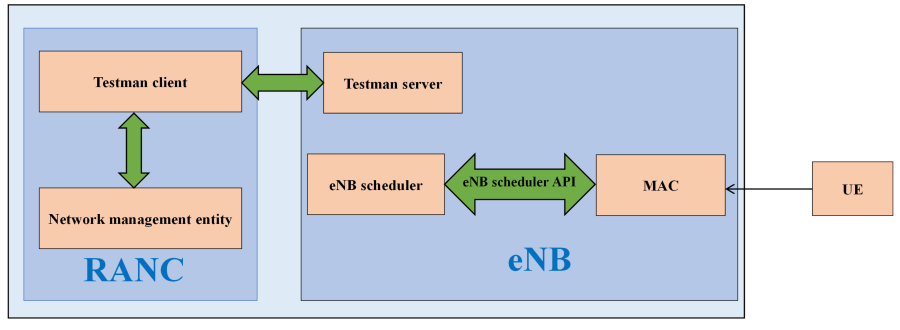

Fig. 4. Experiment design of intelligent switching in multi-RAT framework.

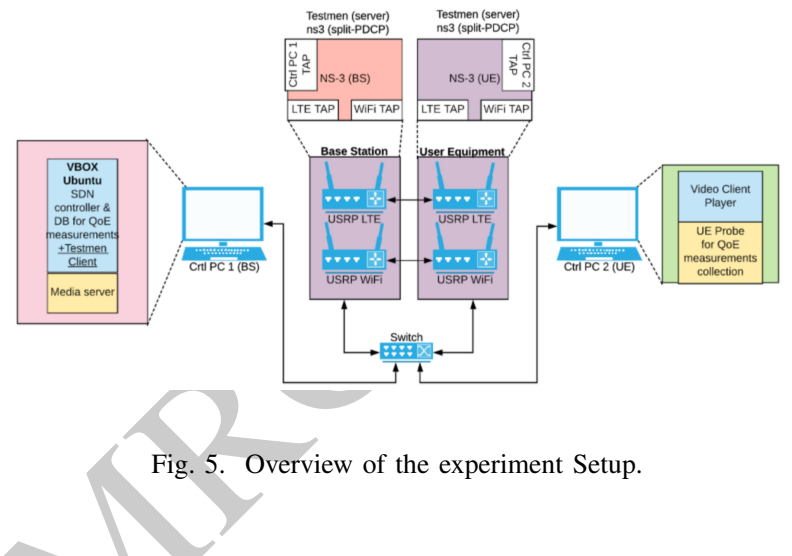

evaluation. Because of the reconfigurable hardware used in the experiment, such implementations can be performed in a software environment where the RANC performs actions based on information sharing with the scheduler. Moreover, we have also conducted another experiment in a multi-RAT environment based on the selecting the modulation and coding scheme (MCS) based on the channel quality indicator (CQI) of LTE links.

\section{A. Deployment of the Implemented Modules}

For the experiments, as shown in Fig. 5 the media server is implemented within the Win7 Host PC connected to the eNB PXI controller whereas the media client is made at the Win7 Host PC connected to the UE PXI controller. The RAN controller modules and the database for the QoE related information exchange are implemented inside the Ubuntu PC. In order to exploit the multi-RAT use case of the testbed, we intend to add noise to the LTE link so that RANC can trigger automatic switching to Wi-Fi link based on the SINR and QoE measurements. For the addition of the noise, we implemented the noise generator using LabVIEW. The noise generator output is combined with the transmission of LTE link using the combiner in the cabling setup to add noise in the LTE downlink channel. The experiment utilizes the NS3 in the TAP bridge configuration which means that external traffic is forwarded to the NS3. Therefore, two packet forwarding scripts are used at PXI controllers: first at eNB PXI controller for forwarding the media traffic from media server into the NS3 TAP bridge and; second at UE PXI controller to forward the media traffic from the NS3 TAP bridge to media client. All the implemented modules are deployed successfully in the testbed except the scheduler module. Though the customised 
scheduler module works fine in the standalone locally installed NI NS3 (simulation mode of NS3 with SDR) but the deployment of the scheduler module into testbed encountered problems using the real physical layer provided by NI LTE Application Framework.

\section{B. Experiment Execution Process}

This section describes the execution process of the proposed experiments. The execution of the experiment followed nine steps in total including the one offline step of data analysis of the collected QoE KPIs from the experiments to acquire results. The process of the experiment execution has following steps:

Step 1: For each scenario, the first step of experiment execution involves the starting the 802.11 Application Framework (needed for Wi-Fi/LWA) and the UE NS3 instance using NI LTE and 802.11 Application Frameworks (AFW). This is performed by compiling the NI NS3 configuration code on the UE controller followed by the starting the transmitter and receiver at UE side.

Step 2 : The second step in the experiment execution involves the compilation of the NI NS3 configuration code at eNB followed by the initiation of the transmitter and receiver at eNB side

Step 3: Once the eNB and UE instances start working, the noise generator at interferer side is configured and initiated to degrade the LTE channel quality of our use case scenario.

Step 4: The media server implemented via VLC is configured for the IPTV video transmission. In this process, a video file is added in the media server for the UDP legacy IPTV streaming which sends the video stream to the eNB PXI controller.

Step 5: In order to forward the video streaming in the NS3 from the media server, video streaming forwarding scripts are used at PXI controller. The forwarding script transfers IPTV video packets to the NS3 TAP bridge which then passes through the NS3 and then over the air interface towards the UE side.

Step 6: The video forwarding script at UE PXI controller is initiated to forward the yideo traffic from NS3 TAP bridge at UE to Win7 host PC host where the media client is running.

Step 7: Depending on the scenario, the RAN controller instance is started. In case of SINR based LWA switching, SINR based RAN controller application is started while in case of the QoE based LWA switching, QoE based RAN controller application is started. In case of only LTE link (baseline case with LWA enabled switching) RAN controller is not initiated.

Step 8: The media client at the Win7 host PC attached to the UE side is started for the IPTV media streaming. Once the media client is started, the UE client side probe implemented in the media client starts sending the QoE KPIs to the database available to the controller.

Step 9: The data analysis step is performed offline. In this step, the data regarding QoE KPIs for all the scenarios is analyzed to acquire the final results.

\section{Performance Analysis}

This section provides a discussion on the achieved results from the proposed experiments. The results provide comparative analysis of multi-RAT scenarios where the LWA based switching is performed by the RANC in case of LTE channel degradation. We have compared the results into different network scenarios.

- Round Robin scheduler in only LTE access network without RAN Controller which is represented as RR-LTE

- The LTE access network only with Proportional Fair scheduler without RAN controller. This scenario is represented as PF-LTE. The SINR based information centric LWA switching from LTE to Wi-Fi using a RANC.

- This is a multi-RAT scenario which is represented as SINR-RANC. The QoE-aware LWA switching from LTE to Wi-Fi via RAN controller

- This scenario is a multi-RAT use case where QoE information is shared from UE to the RAN controller. In the results, this scenario is represented as QoE-RANC

Figure 6 provides a comparison of the four scenarios in terms of accumulated average delivered QoE for all experiments runs in all scenarios. Mean Opinion Score (MOS) is a QoE metric for multimedia (video and audio) traffic ranging scale from 1 (bad) to 5 (excellent). The mean delivered QoE in case of SINR-RANC and QoE-RANC is higher 4.5 MOS while in case of RR-LTE and PF-LTE lower mean QoE is delivered. The higher delivered QoE is the multi-RAT depicting the effectiveness of the information centric RANC to trigger the LWA based switching between LTE and WiFi. Both for the SINR-RANC and the QoE-RANC we can observe that it is delivering almost the same mean QoE for IPTV streaming. This is due to the reason that once the traffic is switched to the Wi-Fi link the channel condition remains the same for both scenarios. Moreover, the time taken by the RANC to enable LWA switching from LTE to Wi-Fi in both cases is more or less the same.

Figure 7 shows the histogram plot of the delivered QoE in all scenarios. The histogram plot for RR-LTE and PF-LTE highlights that the delivered QoE is most frequently in the range of 3.5 and 4.0 MOS respectively. Moreover, the spread of the histogram plot for RR-LTE and PF-LTE scenarios shows that the QoE is not ensured in these scenarios and QoE varies from 1.5-4.6 and 3.0-4.75 MOS respectively. Whereas in SINR-RANC and QoE-RANC, the higher QoE is delivered most often which shows that RAN control usage with the multi-RAT enabling technology ensures the delivered QoE to the user in case of the bad channel conditions. Furthermore, the spread of the histogram for SINR-RANC and QoE-RANC varies from 2.5-4.6 and 3.4-4.8 MOS respectively. According to the study in [11], the frequent changes in delivered quality of the video streaming leads to lower user perceived quality. Therefore, among all the scenarios the QoE-RANC approach outperforms others in terms of the QoE maximization as the QoE-RANC approach delivers higher QoE with less variance. 


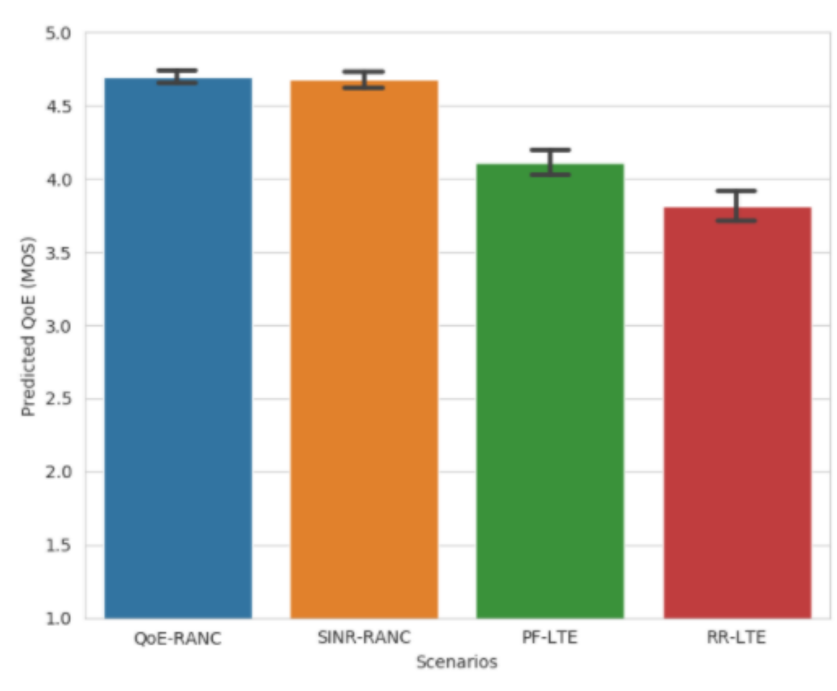

Fig. 6. Accumulated average predicted QoE for each scenario.

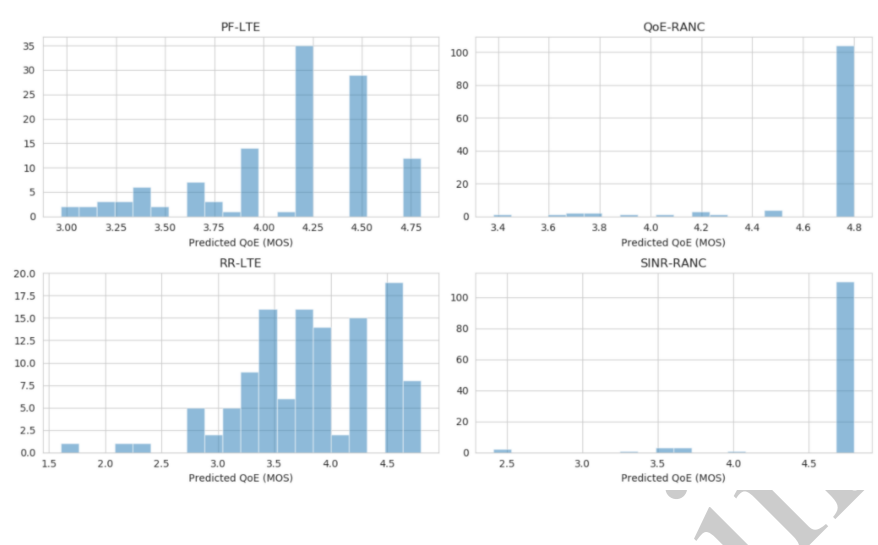

Fig. 7. Histogram plot of predicted QoE (MOS) in each scenario.

\section{CONCLUSiON}

In this paper a prototype solution has been built to test the advantages of a multi-RAT environment controlled by LWA and LWIP mechanisms and the corresponding experiments have been performed. To implement the switching from the two radio access technologies selected, LTE and Wi-Fi, a RANC controller module has been integrated. These experiments clearly show the advantages of the use of LWA and LWIP. A total of four configurations or use cases have been tested, two with single RAT (LTE radio access), but with different scheduling criteria (RR and PF), and two with the two selected RATs (LTE and Wi-Fi), but with different criteria for switching decision (SINR or video QoE). The findings of this research demonstrates that the multi-RAT activation through RANC provides much better QoE for the user compared to a single RAT system. The above-mentioned results indicate that the marriage of SDN-based networks with existing infrastructure of Internet service provider (ISP) is a valuable contribution to traffic steering. Being able to dynamically and adaptively switch traffic between different RATs based on appropriate policies enables new capabilities for handling priority or best effort traffic in collocated networks. The success of such approach will depend on the operators' and infrastructure providers' interest in collaborating with e.g., SDRAN vendors (like 4G, 5G RAN) where the existing capacity of "the pipe" will gain more utilization by availability of OFDMA based "scheduled radio unit" that augments Wi-Fi access points at an indoor location. As a follow up of this research authors foresee the interest in continuing the multiRAT traffic control with the WiFi6 (OFDMA scheduler), 60 GHz links (WiGig), and more than $100 \mathrm{GHz}$ links (Terahertz) for indoor locations.

\section{ACKNOWLEDGMENT}

This work was co-funded by Polish National Centre for Research and Development "Development and implementation of radio access network functionality for future mobile $5 \mathrm{G}$ systems based on cell-free architecture" project (POIR.01.01.0100-0900/17).

\section{REFERENCES}

[1] S. Song, J, Jung, M. Choi, C. Lee, J. Sun and J. Chung, "Multipath Based Adaptive Concurrent Transfer for Real-Time Video Streaming Over 5G Multi-RAT Systems," IEEE Access, pp. 146470-146479, 2019, October 2019.

[2] J. Kim, H. Lee and S. Chong, "Super-MAC Design for Tightly Coupled Multi-RAT Networks," IEEE Trans. Commun., vol. 67, no. 10, pp. 69396951, October 2019.

[3] F. Elsherif and E. K. P. Chong, "Risk-Averse Traffic Allocation for MultiRAT Connectivity in HetNets," 10th Annual Computing and Communication Workshop and Conference (CCWC), 4-8 December6-8 Jan. 2020, Las Vegas, NV, USA, USA, New York: IEEE.

[4] V. F. Monteiro, M. Ericson and F. R. P. Cavalcanti, "Fast-RAT Scheduling in a 5G Multi-RAT Scenario," IEEE Communications Magazine, vol. 55, no. 6, pp. 79-85, June 2017.

[5] M. Anany, M. M. Elmesalawy and A. M. Abd El-Haleem, "Matching Game-Based Cell Association in Multi-RAT HetNet Considering Device Requirements," IEEE Internet of Things Journal, vol. 6, no. 6, pp. 97749782, July 2019.

[6] M. Sepulcre and J. Gozalvez, "A big data deep reinforcement learning approach to next generation green wireless networks," IEEE Trans. on Mobile Computing, 4-8 December 2017, Singapore, pp. 1-1, September 2019.

[7] M. Shahwaiz Afaqui, Cristina Cano, Vincent Kotzsch, Clemens Felber, and Walter Nitzold, "Implementation of the 3GPP LTEWLAN Interworking Protocols in NS-3", Available online: https://arxiv.org/abs/1901.03683.

[8] M. Shahwaiz Afaqui, Cristina Cano, Vincent Kotzsch, Clemens Felber, and Walter Nitzold, "Implementation of the 3GPP LTE-WLAN Interworking Protocols in ns-3", In Proceedings of the 2019 Workshop on $n s-3$ (WNS3 2019). Association for Computing Machinery, New York, NY, USA, 25-32.

[9] M. S. Afaqui, C. Cano, V. Kotzsch, C. Felber and W. Nitzold, "Real-time operation of LTE/Wi-Fi interworking via NS-3 and SDR interfacing", IEEE Conference on Computer Communications Workshops (INFOCOM WKSHPS), Paris, France, 2019, pp. 981-982, doi: 10.1109/INFCOMW.2019.8845047.

[10] [Online], "https://www.orca-project.eu/open-calls/2nd-open-call-forexperiments/".

[11] K. Yamagishi and T. Hayashi, "Parametric Packet-Layer Model for Monitoring Video Quality of IPTV Services", IEEE International Conference on Communications, 19-23 May 2008, Beijing, China.

[12] A. Ahmad, A. Floris and L. Atzori, "Timber: An SDN based emulation platform for QoE Management Experimental Research", The Tenth International Conference on Quality of Multimedia Experience (QoMEX), 29 May-1 June 2018, Cagliari, Italy, 